\title{
O Ensino de Programação Mediado por Tecnologias Educacionais: uma Revisão Sistemática de Literatura
}

\author{
Cleitom José Richter - Instituto Fed. de Educ., Ciênc. e Tecn. Farroupilha (IFFAR) -
} cleitom.richter@iffarroupilha.edu.br

Giliane Bernardi - Programa de Pós-Graduação em Tecnologias Educacionais em Rede - PPGTER - Universidade Federal de Santa Maria (UFSM) - giliane@inf.ufsm.br

Andre Zanki Cordenonsi - Programa de Pós-Graduação em Tecnologias Educacionais em Rede - PPGTER - Universidade Federal de Santa Maria (UFSM) andrezc@inf.ufsm.br

\begin{abstract}
Resumo. Este artigo apresenta os resultados de uma Revisão Sistemática da Literatura (RSL) que buscou investigar e identificar, em nível nacional, as principais tecnologias educacionais e estratégias pedagógicas utilizadas como mediadoras para o ensino de programação de computadores, com ênfase na Programação Orientada a Objetos. Após a seleção dos estudos primários, foram analisados 136 estudos, os quais indicaram que os softwares Scratch e Alice foram os mais citados e as abordagens por Oficinas, Aprendizagem Baseada em Problemas $(P B L)$ e Aprendizagem Baseada em Projetos (ABP) foram as mais exploradas.
\end{abstract}

Palavras-chave: Ensino de Programação. Tecnologia Educacional. Programação Orientada a Objetos.

\section{Educational Technologies and Learning of Programming: a Systematic Literature Review}

\begin{abstract}
This paper presents a Systematic Literature Review (SLR) results which investigates and identify, at national level, the principal educational technologies and pedagogical strategies used as mediators to learning computer programming emphasizing Object Oriented Programming. After the selection of the primary studies, 136 studies were analyzed, which indicated that the software Scratch and Alice were the most cited and the approaches by Workshops, Problems Based Learning and Projects Based Learning were the most explored.
\end{abstract}

Keywords: Learning Programming. Educational Technology. Object Oriented Programming.

\section{Introdução}

As metodologias de ensino e aprendizagem nunca estiveram tão dinâmicas como nos dias atuais. No ensino da computação, a inclusão de tais práticas busca oferecer facilitadores capazes de amenizar as dificuldades de assimilação dos conceitos relacionados à logica computacional. A complexidade relacionada a tais conceitos se deve pelo fato da necessidade de dominar diversas áreas do conhecimento, que perpassam desde a interpretação do problema, o desenvolvimento prático de uma solução e a construção sintática de um algoritmo para tal. Nesse caso, a solução de problemas computacionais desencadeia processos mentais que necessitam da mobilização de habilidades intelectuais diversas. É importante frisar que disciplinas que ensinam Computação são distintas das aulas de Informática, pois agregam conhecimentos e habilidades próprias da ciência que dá origem a ela. Nesse caso, estudar computação possui benefícios educacionais e econômicos, visto que, pelo viés educativo, exercitamos habilidades de reflexão e solução de problemas, pois o mundo está impregnado com a tecnologia digital (BRACKMANN, 2017). 
$\mathrm{Na}$ atualidade, encontramos sérios desafios no ensino da programação de computadores, evidenciados em várias publicações que demonstram que a complexidade dessa área do conhecimento tem ocasionado muitas desistências em diversos cursos no Brasil. Segundo Jesus et al. (2014), as escolas e universidades vêm sofrendo um esvaziamento nos cursos da área de computação tendo em vista a dificuldade dos alunos em compreender a lógica de programação.

Nesse contexto, o presente trabalho tem como objetivo apresentar uma Revisão Sistemática da Literatura (RSL) utilizando a abordagem proposta por Kitchenham (2007), realizada com o objetivo de investigar e identificar quais são as principais estratégias e tecnologias educacionais utilizadas para auxiliar e mitigar os desafios do ensino de programação de computadores, sobretudo no que se refere ao paradigma de Programação Orientada a Objetos (POO), pois considera-se que há pouca diversidade de ferramentas para essa finalidade específica. Embora o objetivo central da RSL foi de investigar o cenário no contexto de POO, considerou-se importante generalizar a pesquisa para todos os paradigmas de programação com o intuito de identificar possíveis estratégias que podem ser utilizadas no cenário específico de POO.

\section{Metodologia da RSL}

Kitchenham (2007) define as diretrizes para a elaboração da RSL a partir da realização de uma sequência de passos que permitem sistematizar todo o processo de revisão. Tal sequência perpassa, primeiramente, pela definição inicial do propósito da pesquisa, o âmbito e o período temporal a ser estudado. Na fase seguinte, é realizada a busca de publicações em fontes de pesquisa determinadas pelo âmbito selecionado. A partir dos resultados brutos dessa fase, é necessário estabelecer critérios de inclusão e exclusão para que sejam selecionadas apenas publicações com potencial para elucidar o objeto de pesquisa. O passo seguinte versa sobre a extração dos dados mais relevantes para a pesquisa. Assim, é necessário estipular critérios de avaliação da qualidade das publicações selecionadas a partir da verificação de seus conteúdos. Por fim, chega-se a parte da análise, em que são tabulados os resultados dos cruzamentos das perguntas de extração. Tal cruzamento é capaz de oferecer as informações necessárias para sintetizar o "estado da arte" do objeto de pesquisa, auxiliando o pesquisador na tomada de decisões quanto ao futuro da pesquisa na área.

\subsection{Objetivo e processo de busca}

Esta revisão tem como objetivo identificar quais são as principais estratégias e tecnologias utilizadas no ensino de programação, realizando uma análise do panorama das pesquisas e experiências no âmbito nacional. Nesse sentido, o trabalho incluiu artigos publicados entre 2010 e 2018. Para essa pesquisa, foram relacionados artigos publicados nos anais dos seguintes eventos e periódicos: Simpósio Brasileiro de Informática na Educação (SBIE); Workshop de Informática na Escola (WIE); Revista Brasileira de Informática na Educação (RBIE); Revista Novas Tecnologias na Educação (RENOTE); Workshop de Ensino em Pensamento Computacional, Algoritmos e Programação (WAlgProg) e Congresso Alice Brasil (Alice). Apesar de não tão conhecido na comunidade, o evento Alice Brasil (realizado entre 2010 e 2016), foi incluído por enfatizar pesquisas envolvendo o paradigma de POO. Já os demais eventos e periódicos foram selecionados pela sua grande relevância no contexto nacional, com contribuições significativas acerca da tríade ensino-tecnologias-computação. 
As questões de pesquisa elaboradas são apresentadas na Tabela 1:

Tabela 1 - Questões de Pesquisa.

\begin{tabular}{ll}
\hline Questões & Descrição \\
\hline QP1 & Que estratégias ou métodos têm sido utilizados para o ensino da programação? \\
QP2 & Quais são os principais softwares utilizados para mediar este processo? \\
QP3 & $\begin{array}{l}\text { Quais benefícios e limitações estão sendo relatados pelo uso das abordagens } \\
\text { propostas para o ensino de POO? }\end{array}$ \\
\hline
\end{tabular}

\subsection{String de busca}

Definidas as questões da pesquisa, procedeu-se a construção de uma string de busca para obtenção de resultados primários dessa RSL. A mesma string é utilizada nos motores de busca de todos os repositórios de dados dos eventos pesquisados. As palavras-chave utilizadas para a sua composição foram: metodologia, ensino e programação. Com base neste conjunto de palavras, a string de busca construída foi: ((ferramenta OR software OR estratégias OR abordagens OR práticas OR metodologias OR métodos) AND ensino AND programação).

\subsection{Condução}

A partir da execução da pesquisa com a string de busca, foram selecionadas 245 publicações para a condução da segunda etapa, na qual foi realizada a leitura dos títulos, resumos e palavras-chave. Esse processo se faz necessário para realizar uma triagem inicial dos trabalhos potenciais para a pesquisa, bem como a exclusão das publicações que não abordam questões relevantes para esta revisão. Nessa fase, foram definidos e aplicados Critérios de Inclusão (CI) e Critérios de Exclusão (CE) para cada estudo. A Tabela 2 relaciona tais critérios:

Tabela 2 - Critérios de inclusão e exclusão

\begin{tabular}{|c|c|}
\hline Critérios de Inclusão & Critérios de Exclusão \\
\hline CI1: É uma ou método de ensino de programação & CE1: Não está em português ou inglês \\
\hline CI2: Usa uma ferramenta especifica & CE2: Anterior a 2010 \\
\hline CI3: Aborda games para o ensino da computação & CE3: Resumos de anais \\
\hline $\begin{array}{l}\text { CI4: Revisão da literatura acerca do ensino da } \\
\text { programação }\end{array}$ & CE4: Não atende aos propósitos da pesquisa \\
\hline
\end{tabular}

Dos 245 estudos iniciais, restaram 136 trabalhos $^{1}$ (55\%). O gráfico da Figura 1 relaciona a distribuição das publicações entre os eventos e o gráfico 2 relaciona a distribuição pelo período investigado.

1 Relação de trabalhos selecionados para extração dos dados disponível em: https://bit.ly/2wlZ4g8. 


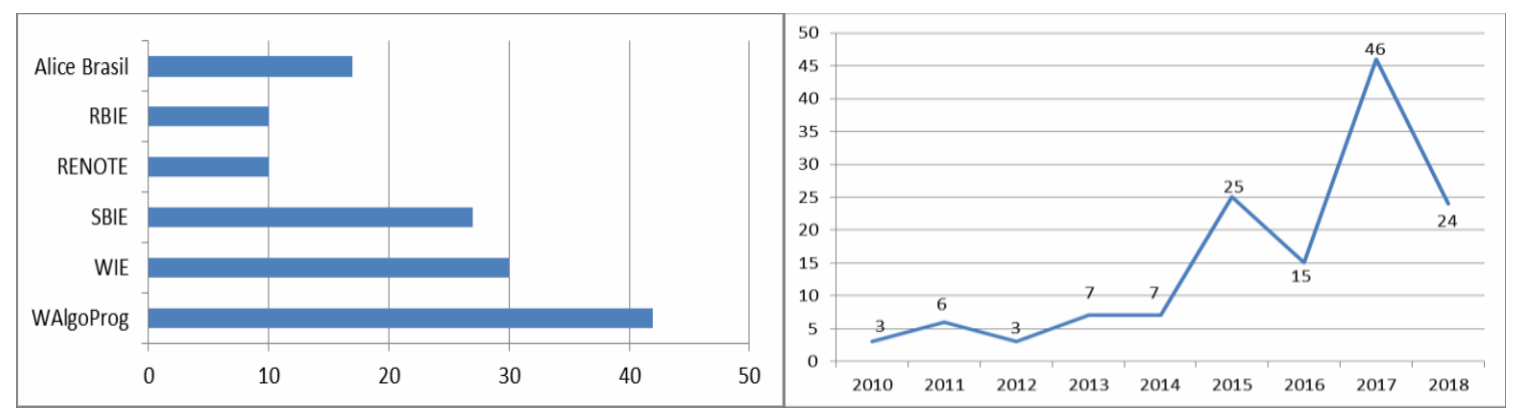

Figura 1. Estudos selecionados por local e por ano de publicação

\section{Resultados e discussões}

Considerando-se que esta pesquisa tinha interesse em identificar os diferentes paradigmas ou níveis de programação envolvidos nos estudos, os mesmos foram classificados e verificou-se que a grande maioria dos trabalhos está relacionada à introdução à programação, pois $83 \%$ dos estudos focaram em Algoritmos ou Lógica de Programação. A Programação Estruturada foi abordada em 15\% dos trabalhos e a Programação Orientada a Objetos em apenas 9,55\%, sendo que vários trabalhos utilizaram abordagens em mais de um nível de programação. As próximas seções respondem individualmente a cada questão de pesquisa.

\subsection{QP1 - Que estratégias ou métodos têm sido utilizadas para o ensino da programação?}

A QP1 tem como objetivo elencar e analisar as principais estratégias e abordagens pedagógicas presentes nos estudos selecionados. Nesse sentido, para obter informações organizadas acerca das estratégias e abordagens verificadas nos estudos, foram definidas categorias que pudessem classificá-las tomando-se como base a proposta de Silva et al. (2015). Assim, a classificação de tais estratégias é apresentada na Tabela 3.

Tabela 3 - Categorias de Tipos de Estudo

\begin{tabular}{|c|c|c|c|}
\hline Abordagem & Qtd & $\%$ & Estudos \\
\hline Avaliação de ferramenta & 4 & $3 \%$ & E19, E21, E25 e E28 \\
\hline Ferramenta de software & 33 & $23 \%$ & $\begin{array}{l}\text { E02, E03, E04, E07, E17, E27, E29, E35, E36, E41, E42, } \\
\text { E54, E61, E62, E64, E67, E72, E73, E74, E79, E83, E84, } \\
\text { E92, E97, E98, E100, E108, E109, E116, E122, E126, E127, } \\
\text { E136 }\end{array}$ \\
\hline Metodologia & 56 & $41 \%$ & $\begin{array}{l}\text { E04, E05, E6, E08, E09, E18, E20, E30, E32, E33, E36, E37, } \\
\text { E39, E43, E44, E48, E49, E51, E52, E54, E56, E57, E58, E59, } \\
\text { E60, E63, E68, E69, E70, E72, E80, E81, E82, E83, E84, } \\
\text { E86, E88, E89, E90, E91, E92, E101, E102, E103, } \\
\text { E104, E111, E114, E116, E118, E119, E122, E125, E127, } \\
\text { E130, E131, E132 }\end{array}$ \\
\hline Robótica & 19 & $13 \%$ & $\begin{array}{l}\text { E06, E10, E14, E16, E18, E33, E55, E56, E60, E69, E80, } \\
\text { E81, E85, E87, E99, E101, E123, E129, E130 }\end{array}$ \\
\hline Games & 24 & $17 \%$ & $\begin{array}{l}\text { E11, E12, E13, E15, E19, E31, E32, E34, E47, E59, E63, } \\
\text { E82, E95, E96, E102, E103, E104, 106, E112, E115, E119, } \\
\text { E124, E128, E133 }\end{array}$ \\
\hline
\end{tabular}

Silva et al. (2015) sugere, ainda, a existência de outras classificações, porém nos estudos pesquisados nessa revisão sistemática não foram observados em sua totalidade. Constatou-se que 3\% dos estudos destinou-se a realização de avaliações de ferramentas 
de software. Nesse caso, os softwares avaliados foram: Lord of code, RHODES 2.0, iVProg e Scratch.

O uso da robótica e de games esteve presente em, respectivamente, $13 \%$ e $17 \%$ dos estudos, sendo que alguns destes apareceram em mais de uma categoria, pois foram desenvolvidos utilizando games ou robótica em conjunto com alguma metodologia - é o caso dos estudos E56, E59, E60 e E63. O desenvolvimento de softwares para apoiar o ensino de programação esteve presente em $23 \%$ dos estudos pesquisados.

Metodologias ou estratégias pedagógicas diversas foram relatadas em $41 \%$ dos trabalhos (Tabela 4):

Tabela 4 - Metodologias e ou estratégias pedagógicas relatadas

\begin{tabular}{lll}
\hline Descrição & Qtd & Estudos \\
\hline Oficinas & & E59, E60, E63, E84, E88, E89, E90, \\
& 16 & $\begin{array}{l}\text { E91, E111, E114, E119, E125, } \\
\text { E127, E130, E131, E132 }\end{array}$ \\
\hline Aprendizagem baseada em projetos & 10 & $\begin{array}{l}\text { E33, E43, E44, E48, E49, E51, E52, } \\
\text { E68, E69, E83 }\end{array}$ \\
Aprendizagem baseada em problemas & 8 & E05, E18, E39, E56, E86, E116, \\
Gamificação & 4 & E82, E102, E103, E104 \\
Objetos de aprendizagem & 3 & E04, E06, E58 \\
Competição/Olimpíada & 3 & E09, E32, E70 \\
Pesquisa-Ação & 3 & E80, E92, E101 \\
Sala de aula invertida & 2 & E54, E57 \\
Ensino Híbrido & 2 & E20, E72 \\
Outros (citado apenas uma vez) & 6 & E8, E30, E36, E37, E39, E81 \\
\hline
\end{tabular}

\subsection{QP2 - Quais são os principais softwares utilizados para mediar este processo?}

Para responder a QP2 procurou-se listar todas as ferramentas de software cuja utilização foi explorada para facilitar o ensino e aprendizagem de programação de computadores nos estudos pesquisados. Desse modo, constatou-se que 130 dos trabalhos utilizaram um ou mais tipos de ferramenta para auxiliar no ensino de programação, sendo que a lista e quantitativos estão discriminados na Tabela 5.

Tabela 5 - Ferramentas de software

\begin{tabular}{llll}
\hline software & $\mathrm{N}^{\mathrm{o}}$ publicações & $\%$ & Estudos \\
Scratch & 22 & & $\begin{array}{l}\text { E2, E3, E4, E7, E8, E9, E11, E15, E24, E28, } \\
\text { E29, E47, E50, E60, E61, E63, E65, E68, E83, } \\
\text { E88, E90, E120 }\end{array}$ \\
Alice & 16 & $19,8 \%$ & $\begin{array}{l}\text { E38, E39, E40, E41, E42, E43, E44, E45, E46, } \\
\text { E48, E49, E50, E51, E52, E53, E65 }\end{array}$ \\
LEGO® Mindstorms & 11 & $14,4 \%$ & E6, E10, E56, E57, E60, E65, E69, E81, E89, \\
MIT AppInventor & 5 & $10 \%$ & E125, E130 \\
Robocode & 3 & $4,5 \%$ & E17, E65, E91, E122, E117 \\
Construct 2 & 3 & $2,7 \%$ & E32, E95, E99 \\
Scratch for Arduino & 3 & $2,7 \%$ & E100, E115, E119 \\
CFácil & 3 & $2,7 \%$ & E33, E55, E87 \\
Arduino IDE & 3 & $2,7 \%$ & E35, E64, E108 \\
Moodle & 2 & $2,7 \%$ & E16, E33, E101 \\
\hline
\end{tabular}




\begin{tabular}{llll}
\hline GameMaker Studio & 2 & $1,8 \%$ & E13, E133 \\
Blockly & 2 & $1,8 \%$ & E27, E54 \\
FARMA-ALG & 2 & $1,8 \%$ & E62, E98 \\
CodeBench (Juíz online) & 2 & $1,8 \%$ & E20, E72 \\
& & E12, E25, E14, E18, E19, E21, E33, E54, E73, \\
Outros (citado uma vez) & 33 & E74, E79, E67, E92, E93, E84, E80, E97, E96, \\
& & $29,7 \%$ & E99, E102, E104, E106, E107, E126, E127, \\
& & E128, E129, E136, E112, E109, E116, E131, \\
\hline
\end{tabular}

Nesse caso é importante observar que duas ferramentas obtiveram destaque nos trabalhos pesquisados, Scratch $(19,8 \%)$ e Alice $(14,4 \%)$, todavia, vale ressaltar que dos estudos que citam Alice, 14 dos 16 foram selecionados a partir do evento Alice Brasil, cuja ênfase é dada a praticas que abordam o uso de dessa ferramenta para o ensino de programação.

\subsection{QP3 - Quais benefícios e limitações estão sendo relatados pelo uso das abordagens propostas para o ensino de POO?}

Dos resultados encontrados, apenas 12 exploraram o ensino de POO em sua abordagem, E1, E19, E32, E39, E42, E43, E44, E48, E51, E112, E113, E118, E132 cujo detalhamento está representado pela Tabela 6.

Tabela 6 - Detalhamento dos estudos que abordam POO

\begin{tabular}{llll}
\hline Critério extração & & Qtd & Estudo \\
\hline É uma RSL & & 1 & E1 \\
Foi testado em sala de aula & 10 & E19, E39, E43, E44, E48, E51, E112, \\
& Ensino Fundamental & 1 & E43 \\
Nível de ensino & Ensino Médio & 1 & E32 \\
& Ensino Técnico & 3 & E112, E113, E118 \\
& Ensino Superior & 5 & E19, E39. E44, E51, E132 \\
\multirow{5}{*}{ Software } & Alice & 6 & E39, E42, E43, E44, E48, E51 \\
utilizado & Lord of Code & 1 & E19 \\
& PPlay & 1 & E112 \\
& JES e Python & 1 & E113 \\
& Xamarin Forms & 1 & E132 \\
& Robocode & 1 & E32 \\
& Aprendizagem baseada em & 4 & E43, E44, E48, E51 \\
& projetos & & \\
Aprendizagem baseada em & 3 & E39, E44, E118 \\
problemas & 3 & E19, E32, E112 \\
Estratégias & Games & 2 & E112, E113 \\
& Manipulação de mídias & 1 & E39 \\
& Mapas conceituais & 1 & E132 \\
& Oficinas & 1 & E19 \\
& Avaliação de ferramenta & & \\
\hline
\end{tabular}

A partir desse detalhamento, constatou-se que a grande maioria dos trabalhos foram testados em sala de aula e que a maior parte desses estão direcionados para o ensino superior ou técnico. Alice foi a ferramenta de software mais utilizada e Aprendizagem por Projetos, Aprendizagem Baseada em Problemas e Games as metodologias e ou estratégias mais evidenciadas.

Para responder a QP3, além da análise quantitativa, se realizou a exploração qualitativa desses estudos. No único trabalho de cunho teórico [E1], os autores 
(SOUZA, BATISTA, BARBOSA, 2016), concluem que as principais dificuldades dos alunos são: aprender os conceitos de programação; aplicar esses conceitos durante a construção de programas; falta de motivação para a realização da atividade de programação. Para amenizar tais problemas e dificuldades, eles elencam possíveis caminhos: utilização de visualização de programas e algoritmos; uso de games; desenvolvimento de ambientes pedagógicos para o ensino e aprendizagem de programação.

Souza et al. (2016), no estudo E19, comentam sobre a dificuldade dos estudantes em abstrair a aprendizagem de programação, pois é um processo complexo que envolve habilidades diversas exigindo alta carga cognitiva para que estes obtenham sucesso. Os autores destacam ainda que, nos cursos de computação, as disciplinas que envolvem programação de computadores são as que apresentam maiores índices de desistência ou reprovação. Nesse trabalho, é proposto o desenvolvimento do ambiente Lord of Code, baseado na utilização de jogos para estimular a aprendizagem de programação. Uma avaliação piloto foi realizada com alunos de graduação em Licenciatura em Computação do IFMG - campus Ouro Preto, onde a maioria avaliou a ferramenta entre "bom" e "poderia ser melhor".

Semelhante a essa experiência, Amaral et al. (2015)[E32] utilizaram a ferramenta Robocode ${ }^{2}$ para organizar uma olimpíada de programação envolvendo desafios gamificados com estudantes do ensino médio de escolas públicas da cidade de Patos de Minas/MG. Essa experiência consistiu na organização de um campeonato de programação em que os participantes deveriam programar um robô com funcionalidades para combater em um cenário de guerra. Quanto mais aprimoradas fossem as funcionalidades desenvolvidas para esse robô, maiores seriam as chances de sucesso na competição. Nesse caso, o fator determinante da atividade envolve agilidade, pensamento computacional e conhecimento em linguagem de programação - que, no caso da ferramenta, é orientada a objetos.

Outro caso de sucesso do uso de ferramentas para o ensino de POO é relatado por Antonio e Ferro (2011) [E39], que abordam a utilização do ambiente Moodle, a técnica de aprendizagem baseada em problemas, o ambiente de programação Alice 3D e mapas conceituais. A experiência relatada apresentou bons resultados no que tange a pratica de ensino de POO, visto que a metodologia consistiu na aplicação de um questionário antes do desenvolvimento da dinâmica e depois de concluído o processo. Os autores destacam ainda a importância do software utilizado, enfatizando que "o uso do software Alice, despertou o interesse e facilitou o entendimento desses conceitos".

Craveiro et al. (2011) [E42] destacam as funcionalidades do software Alice para o ensino de POO, pois consiste em um ambiente lúdico de desenvolvimento no qual é possível trabalhar a modelagem de classes de maneira diferenciada do que normalmente se trabalha nas escolas, pois em cada ator do cenário (objeto) é possível a configuração de características (atributos) e ações (métodos) próprias. Outro trabalho que utiliza o software Alice é apresentado em Ferreira (2012) [E43], para promover um festival de animação para estudantes das séries finais do Ensino Fundamental. O referido festival foi chamado de Animalice, inspirado no festival "Anima Mundi", e consiste em uma

\footnotetext{
${ }^{2}$ Link: http://www.robocodebrasil.com.br/

${ }^{3}$ O Festival Internacional de Animação do Brasil, ou Anima Mundi, é uma organização de animações que ocorre anualmente no mês de julho nas cidades do Rio de Janeiro e São Paulo, no Brasil.
} 
competição que envolve as disciplinas de Robótica, Programação e temas interdisciplinares.

Antonio e Ferro (2013) [E44] utilizaram o software Alice no ensino de programação para estudantes de Sistemas de Informação. A dinâmica consistiu na realização da sequência de cinco atividades em que uma era pré-requisito para a próxima. Ao final da experiência, os autores destacam que os resultados são:

[...] promissores na tentativa de inserir o software Alice como parte das atividades didáticas pedagógicas para o ensino de programação. Os resultados encontrados, especialmente do ponto de vista da avaliação qualitativa da aplicação das atividades ao longo do semestre, permitiram evidenciar a motivação dos alunos ao elaborarem as atividades sugerindo a real viabilidade de aplicação do software no contexto educacional do curso de Sistemas de Informação (ANTONIO E FERRO, 2013).

Andrade et al. (2016) [E51] seguem a mesma linha ao proporem o desenvolvimento de uma atividade que, através da construção de um cenário no software Alice, permitia que o estudante explorasse os conceitos básicos de $\mathrm{OO}$ de maneira lúdica. Essa atividade foi desenvolvida com estudantes do ensino superior que estão cursando a disciplina de Análise, Projeto e Desenvolvimento de Sistemas. A partir da "percepção qualitativa", as autoras relatam que a atividade proposta permitiu a aplicação de técnicas ativas de aprendizagem, explorando a proatividade dos estudantes na elaboração de projetos e na aplicação de conceitos básicos de $\mathrm{OO}$ de maneira lúdica.

No trabalho de Ribeiro et. al (2017) [E113], o ensino de POO é abordado a partir da manipulação de imagens, utilizando a ferramenta JES (Jython Environment for Students), que utiliza Python. Pontos positivos foram observados no que se refere a facilidade de compreender os conceitos de POO pela visualização instantânea dos resultados da programação e pela aproximação do objeto estudado ao contexto dos alunos. A mesma ferramenta é explorada em Araujo et. al. (2017) [E112] para manipulação de imagens, e a ferramenta PPlay para o desenvolvimento de jogos. Através dessa experiência, os autores puderam constatar que Python é adequado aos estudantes iniciantes, pois oferece facilidades na compreensão dos conceitos de programação e POO. Ainda, que a realização de atividades contextualizadas gera bons resultados no que se refere ao engajamento dos alunos. Constatou-se, também, que o uso de desafios pode ser positivo, pois os estudantes sentem-se motivados a alcançar o objetivo final de algo que construíram por esforço próprio. Como fragilidades da pesquisa, os autores destacam o reduzido esforço em estudos extra-classe por parte dos alunos.

Gonçalves et al. (2017) [E118] desenvolvem um trabalho com estudantes do ensino profissionalizante, abordando a aprendizagem dos conceitos de POO pela estratégia Aprendizagem Baseada em Problemas. Por meio desta experiência, foi possível perceber benefícios no que se refere à motivação e autonomia dos estudantes por utilizar uma metodologia ativa de ensino; e fragilidades, tendo em vista que alguns estudantes se sentiram perdidos ou excluídos das atividades.

Por fim, Beltrame (2018) [E132] propõe uma dinâmica de ensino, para estudantes de um curso de graduação em Engenharia Elétrica baseada na implementação de soluções para problemas reais dos aprendizes, fundamentando-a na construção de aplicativos para dispositivos móveis. Como resultados, evidenciou-se a motivação dos estudantes com a proposta; em contrapartida, ao usar de várias ferramentas para 
desenvolver a proposta, houve um grande esforço de configuração das mesmas, gerando descontentamento dos alunos.

\section{Considerações Finais}

Esse artigo apresentou uma Revisão Sistemática da Literatura sobre estratégias e tecnologias educacionais utilizadas para o ensino de programação de computadores entre os anos de 2011 e 2018. De um total de 245 trabalhos pré-selecionados, 136 foram tabulados e avaliados e 12 são discutidos, centrando-se naqueles que abordaram o paradigma de POO.

Em relação ao ensino de programação, percebeu-se que o Scratch e o Alice foram os softwares mais utilizados. O primeiro, baseado no ensino através de blocos de programação, foi amplamente utilizado com alunos do ensino fundamental e médio, além de experimentos nos anos iniciais dos cursos técnico e superior. $\mathrm{O}$ Alice, por sua vez, tem como arquitetura a orientação a objetos e, por isso, seu uso é mais amplo quando POO era o objetivo do estudo. Além disso, percebe-se que a estratégia de ensino através de oficinas se mostrou bastante presente, seguida rapidamente pelas metodologias ativas de aprendizagem baseada em problemas ou projetos. Esta constatação parece indicar que os pesquisadores estão preocupados em implementar estratégias voltadas a uma aproximação do aluno com a sua realidade, bem como buscando uma maior autonomia dos mesmos. Isso poderia mitigar os diversos problemas levantados nos trabalhos pesquisados, que colocam a dificuldade de compreensão dos aspectos abstratos como uma das dificuldades dos alunos. A robótica e a utilização dos games (utilizados em 19 e 24 estudos, respectivamente), também parecem indicar este caminho.

Em relação à POO, a escassez dos estudos selecionados talvez possa ser explicada pela ampliação das questões relacionadas com o Pensamento Computacional e a inclusão do mesmo nos currículos escolares proposto por vários autores, pois boa parte dos esforços têm se centrado no ensino de lógica de programação e o paradigma estruturado. No entanto, o paradigma possui uma estrutura lógica bastante diversa e apenas a aprovação em disciplinas anteriores de programação não é o suficiente para que o aluno compreenda corretamente os detalhes basilares da POO. Nos estudos analisados, a maior parte apresenta como justificativa a utilização de estratégias diversas para melhorar o entendimento dos conceitos fundamentais do paradigma, como classes, heranças e polimorfismo.

Desta forma, conclui-se que há existência de um espaço a ser melhor explorado pela produção de referencial e métodos que auxiliem tais práticas. A reunião de estratégias que unam uma aproximação do aluno com a sua realidade e a utilização de tecnologias diversas, como games ou ferramentas de software, parece ser a melhor abordagem para estudos futuros.

\section{Referências}

AMARAL, L. R.; BRAGA E SILVA, G.; PANTALEÃO, E. Plataforma Robocode como Ferramenta Lúdica de Ensino de Programação de Computadores - Extensão Universitária em Escolas Públicas de Minas Gerais. In: Anais do SBIE, 2015.

ANDRADE, K. C. R.; MORENO, R.; ROSSI, A. C. Experiência de uso do software Alice em uma atividade de análise, projeto e desenvolvimento de sistemas aplicando os conceitos básicos de orientação a objetos. In: Anais do Alice Brasil, 2016. 
ANTONIO, E. A., FERRO, M. Avaliação do software Alice 3D para o Ensino de Orientação a Objetos apoiada por Ambientes Virtuais, PBL e Mapas Conceituais. In: Anais do Alice Brasil, 2011.

- O uso do Software Alice 3D como Motivação para o EnsinoAprendizado de Programação: Exemplos de Atividades para o Curso de Sistemas de Informação. In: Anais do Alice Brasil, 2013.

ARAUJO, L. G. de J.; BITTENCOURT, R. A.; SANTOS, D. M. B. dos. Uma Abordagem Contextualizada para o Ensino de Programação na Educação Profissional em Informática. In: Anais dos WCBIE, 2017.

BELTRAME, W. A. R. Projeto Ágil de Aplicativo como Mediação da Aprendizagem sobre Orientação a Objetos. In: Anais do WCBIE, 2018.

BRACKMANN, C. P. Desenvolvimento do pensamento computacional através de atividades desplugadas na educação básica. 2017. 226f. Tese (Doutorado em Informática na Educação) - UFRGS, Porto Alegre, 2017

CRAVEIRO, M. V. et al. Utilizando o software ALICE como apoio para o Ensino de Lógica de Programação - Parte 3: Programação Orientadas a Objetos. In: Anais do Alice Brasil, 2011.

FERREIRA, F. Animalice - Festival de Animação do Alice: o uso do software Alice como ferramenta de aprendizagem na disciplina de robótica para estudantes do Ensino Fundamental. In: Anais do Alice Brasil, 2012, São Paulo.

GONÇALVES, M. D. et al. Percepções Sobre Metodologias Ativas de Aprendizagem de Programação no Ensino Profissionalizante. In: Anais do WCBIE, 2017.

JESUS, A. M.; GONÇALVES, D. A. S.; FERREIRA, L. A. C. Aplicação de Desenvolvimento de Jogos Digitais como um Meio de Motivação em Diferentes Níveis de Ensino de Computação. In: Anais do WIE.

KITCHENHAM, B. Guidelines for performing Systematic Literature Reviews in Software Engineering, version 2.3. Technical Report EBSE. Software Engineering Group. School of Computer Science and Mathematics Keele University. 2007.

RIBEIRO, A. L. et al. Computação com Mídias na Aprendizagem de Programação Orientada a Objetos em um Curso Técnico de Informática. In: Anais do WCBIE, 2017.

SILVA et al. Ensino-aprendizagem de programação: uma revisão sistemática da literatura. In: RBIE, v. 23, n. 1, 2015.

SOUZA, D. M.; BATISTA, M. H. S.; BARBOSA, E. P. Problemas e Dificuldades no Ensino e na Aprendizagem de Programação: Um Mapeamento Sistemático. In: RBIE, v.24, n.1, 2016.

SOUZA, M. S. C; COSTA, F. A M; SILVA, V. L.; TERRA, D. C. Lord of Code: uma ferramenta de apoio ao ensino de programação. In: Anais do SBIE, 2016.

VALENTE, J. A. Integração do Pensamento Computacional no Currículo da Educação Básica: Diferentes Estratégias Usadas e Questões de Formação de Professores e Avaliação do Aluno. In: e-Curriculum, v. 14, n. 3, 2016. 